\title{
Maladies bactériennes invasives dans le Nord du Canada de 2006 à 2013
}

\author{
Li YA ${ }^{1 *}$, Martin I², Tsang R2 , Squires SG ${ }^{1}$, Demczuk W², Desai $\mathrm{S}^{1}$
}

\section{Résumé}

Contexte : Les populations du Nord présentaient un risque plus élevé de contracter des maladies bactériennes invasives (MBI) comparativement à celles du reste du Canada. Depuis la dernière étude publiée sur les maladies bactériennes invasives dans le Nord du Canada, un certain nombre de vaccins contre des pathogènes bactériens ont été introduits au calendrier de vaccination systématique des enfants.

Objectif : Décrire l'épidémiologie des maladies bactériennes invasives dans le Nord du Canada de 2006 à 2013 et comparer leurs incidences dans le reste du pays.

Méthodologie : Les données relatives à cinq maladies bactériennes invasives (pneumococcie invasive [PI], infection à Haemophilus influenzae [Hi], infection invasive à streptocoques du groupe $\mathrm{A}[\mathrm{SAl}]$, méningococcie invasive $[\mathrm{MI}]$ et infection invasive à streptocoques du groupe $\mathrm{B}$ [SBI]) ont été tirées du programme du Système international de surveillance circumpolaire (SISC) et du Système canadien de surveillance des maladies à déclaration obligatoire. Les taux d'incidence sont calculés pour 100000 habitants par an.

Résultats : Durant la période de l'étude, les taux d'incidence variaient comme suit : PI : de 16,84 à 30,97 ; SAI : de 2,70 à 17,06; Hi de sérotype $b$ : de 0 à 2,78; Hi de type non-b : de 2,73 à 8,53 et $\mathrm{Ml}$ : de 0 à 3,47 pour 100000 habitants. À l'exception de la méningococcie invasive et de l'infection invasive à streptocoques du groupe $B$, les taux d'incidence standardisés en fonction de l'âge des autres maladies dans le Nord du Canada étaient de 2,6 à 10 fois plus élevés que dans le reste du pays. Au cours de la période de l'étude, les taux ont baissé dans le cas de pneumococcie invasive $(p=0,04)$ et de l'infection invasive à streptocoques du groupe $\mathrm{A}(p=0,01)$ et ont augmenté dans le cas de l'infection à Hi de type a (Hia) $(p=0,004)$. Parmi les cas de pneumococcie invasive, la proportion des sérotypes du vaccin conjugué antipneumococcique heptavalent a diminué $(p=0,0004)$ pendant la période de l'étude. Parmi les cas d'infection à $\mathrm{Hi}, 69,8 \%$ étaient des cas d'infection à Hia et 71,6\% de celles-ci ont été déclarées chez des enfants de moins de cinq ans. Des 13 cas de méningococcie invasive, huit étaient du sérogroupe B et deux en sont décédés. Dans le Nord du Canada, I'incidence de PI, de SAl et de Hi était de 2,6 à 10 fois plus élevée que dans le reste du pays.

Conclusion : La population du Nord du Canada, particulièrement les nourrissons et les personnes âgées des Premières Nations, court un risque plus élevé de contracter une pneumococcie invasive, une infection à $\mathrm{Hi}$ et une infection invasive à streptocoques du groupe A que la population non autochtone. L'infection à Hi est le sérotype prédominant dans le Nord du Canada.

\section{Affiliations}

'Direction générale de la prévention et du contrôle des maladies infectieuses, Agence de la santé publique du Canada, Ottawa (Ontario)

${ }^{2}$ Laboratoire national de microbiologie, Agence de la santé publique du Canada, Winnipeg (Manitoba)

*Correspondance : anita.li@phacaspc.gc.ca

Citation proposée : Li YA, Martin I, Tsang R, Squires SG, Demczuk W, Desai S. Maladies bactériennes invasives dans le Nord du Canada de 2006 à 2013. Relevé des maladies transmissibles au Canada 2016;42:84-91. https://doi.org/10.14745/ccdr.v42i04a01f

\section{Introduction}

Établi en 1999, le Système international de surveillance circumpolaire (SISC) est un programme qui consiste en un réseau de surveillance fondée sur la population de pays circumpolaires, dont les États-Unis, le Canada, le Groenland, I'Islande, la Norvège, la Suède, la Finlande et la Russie (1). Au Canada, les régions du Nord (Yukon, Territoires du Nord-Ouest, Nunavut, Labrador ainsi que la nation Cri et la région du Nunavik au Québec) et un réseau de laboratoires (le Centre national pour le streptocoque [CNS] [1999 à 2009]), le Laboratoire de santé publique du Québec et le Laboratoire national de microbiologie (LNM) ont participé au programme international de surveillance circumpolaire. Le programme surveille l'infection invasive causée par Streptococcus pneumoniae (pneumococcie invasive [PI]) depuis 1999 et les infections invasives causées par Streptococcus pyogenes (infection invasive à streptocoques du groupe A [SAI]), Streptococcus agalactiae (infection à streptocoques du groupe B [SBI], Haemophilus influenzae $(\mathrm{Hi})$ 
et Neisseria meningitidis (pneumococcie invasive (MI) depuis I'an 2000.

La démographie du Nord du Canada diffère du reste du pays. En 2013, la population du Nord du Canada était estimée à 155666 habitants, ce qui représente 0,4\% de la population canadienne. Néanmoins, la proportion des habitants qui se reconnaissent comme Autochtones (Premières Nations, Métis ou Inuits) était d'environ $60 \%$ comparativement à environ $4 \%$ dans l'ensemble du Canada. Les populations nordiques, et plus particulièrement les personnes autochtones, présentent des taux plus élevés de maladies bactériennes invasives (MBI) comparativement aux populations du reste du Canada (2-6).

La dernière étude publiée qui décrivait les maladies bactériennes invasives dans le Nord du Canada comprenait les données de 1999 à 2005 (5). Depuis, un certain nombre de vaccins contre certains pathogènes bactériens ont été introduits au calendrier de vaccination systématique des enfants. Au Canada, le Comité consultatif national de l'immunisation (CCNI) recommande les vaccins et leur calendrier de vaccination, mais la mise en œuvre des programmes d'immunisation varie d'une province ou d'un territoire à un autre. Dans le cas de la pneumococcie invasive, les programmes d'immunisation systématique à l'aide du vaccin conjugué antipneumococcique heptavalent (VCP7) ont commencé en 2002 et étaient pleinement mis en œuvre dans tout le Nord du Canada en janvier 2006 (7). En 2010, les programmes d'immunisation contre la pneumococcie invasive ont commencé à remplacer le VCP7 par le vaccin décavalent (VCP10) conjugué contre le pneumocoque 10-valent. En janvier 2011, le programme d'immunisation des enfants contre la pneumococcie invasive de l'ensemble des six régions prescrivait le vaccin 13-valent (VCP13) conjugué contre le pneumocoque. Le vaccin polysaccharidique 23-valent contre le pneumocoque (Pneu-P-23) est utilisé chez les populations cibles comme les personnes âgées de 65 ans et plus et les personnes qui présentent un risque élevé de contracter une pneumococcie invasive (8). Les programmes d'immunisation systématique des enfants contre l'infection à $\mathrm{Hi}$ de type b sont mis en œuvre depuis 1997 (8). Pour ce qui est de la méningococcie invasive, les programmes d'immunisation systématique des enfants à l'aide du vaccin conjugué contre le méningocoque du groupe $C$ ont été mis en œuvre dans l'ensemble des six régions en 2007.

L'objectif du rapport est de décrire l'épidémiologie des maladies bactériennes invasives dans le Nord du Canada de 2006 à 2013 et de comparer l'incidence de ces maladies à celle du reste du pays.

\section{Méthodologie}

\section{Données épidémiologiques}

Les données de surveillance pour le Nord du Canada et pour le reste du pays ont été tirées du SISC et du Système canadien de surveillance des maladies à déclaration obligatoire (SCSMDO), respectivement, portant sur l'apparition de la maladie entre

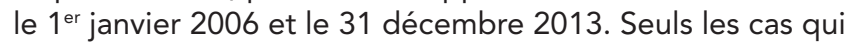
répondaient aux définitions de cas nationales (10) ont été pris en compte. Les coordonnateurs régionaux du SISC ont rempli des formulaires pour la surveillance d'infections bactériennes particulières lorsque les cas répondent aux définitions de cas nationales (10), puis ont colligé et examiné les données de laboratoire. Les données consignées dans les formulaires pour la surveillance des infections bactériennes sont notamment les renseignements démographiques, les renseignements cliniques, les résultats, les facteurs de risque et les antécédents vaccinaux. les formulaires et les rapports de laboratoire sont envoyés à l'Agence de la santé publique du Canada selon un processus sécurisé. Une fois l'an, le SCSMDO reçoit les données agrégées contenant les renseignements démographiques non nominatifs des provinces et des territoires.

\section{Données de laboratoire}

Des isolats invasifs ont été soumis au LNM, au Centre national pour le streptocoque (CNS) [de 2006 à 2009] ou au Laboratoire de santé publique du Québec. Le sérotypage de S. pneumonia au moyen de la réaction Quellung a été effectué au moyen d'antisérums commerciaux polyvalents, de groupe, de type et de facteur de SSI Diagnostica - Statens Serum Institut, de Copenhague, au Danemark $(11,12)$. Les types de séquence du gène emm pour les isolats de l'infection invasive à streptocoques du groupe $A$ ont été déterminés selon la méthodologie recommandée par les Centers for Disease Control and Prevention (CDC) des États-Unis (13). Les sérotypes du streptocoque du groupe B causant une infection invasive ont été déterminés au moyen d'antisérums commerciaux d'agglutination au latex de SSI Diagnostica $(11,12)$. Le sérotypage de $H$. influenzae a été effectué au moyen d'un test d'agglutination des bactéries à l'aide d'antisérums de Difco Laboratories (BD Diagnostics, Falcon Lakes, New Jersey, États-Unis), et les résultats ont été confirmés par réaction en chaîne de la polymérase (14). Les souches non typables de Hi ont été confirmées par séquençage de l'ARN ribosomal 16S (15). Le sérogroupage de $N$. meningitidis a été effectué selon les méthodes d'agglutination des bactéries (16). Tous les laboratoires cités participent à un programme de contrôle de la qualité du SISC (17).

\section{Données démographiques}

Les estimations de la population générale ont été obtenues de Statistique Canada (18). Puisque Statistique Canada ne fournit que les estimations de la population autochtone pour les années de recensement 2006 et 2011, les estimations agrégées de la population autochtone (Premières Nations, Métis ou Inuits) du présent rapport ont été obtenues des divisions des statistiques territoriales ou régionales. Les estimations de la population autochtone du Labrador et de la population Crie au Québec n'ont pu être obtenues qu'à partir des données des recensements de 2006 et de 2011. Les estimations de la population de chaque groupe autochtone n'étaient pas disponibles pour ce rapport. La population canadienne de 1991 a été choisie comme population stable pour la standardisation de l'âge. La répartition de la population est fondée sur les estimations finales de la population canadienne post-recensement du $1^{\text {er }}$ juillet 1991, ajustée en fonction du sous-dénombrement du recensement. La répartition selon l'âge de la population a été pondérée et normalisée (19).

Les données utilisées dans ce rapport ont été tirées de la surveillance de la santé publique, et ont été dispensées de l'approbation du comité d'examen éthique. 


\section{Analyse}

Les données démographiques, les répartitions de sérotypes de même que les caractéristiques cliniques et l'état vaccinal des cas atteints d'une maladie bactérienne invasive ont été examinés. Les taux d'incidence de streptocoque du groupe B chez les nouveau-nés n'ont pas été calculés parce que les estimations de naissances d'enfants vivants dans les régions du Nord n'étaient pas disponibles pour ce rapport. Tous les taux d'incidence ont été calculés pour 100000 habitants par année. La méthode directe a été utilisée pour le calcul des taux standardisés en fonction de l'âge. Les intervalles de confiance (IC) des taux standardisés en fonction de l'âge ont été calculés selon la méthode fondée sur la distribution gamma (20). Les cas dont l'âge n'était pas déterminé ont été exclus du calcul des taux standardisés en fonction de l'âge. Le test chi carré et la méthode exacte de Fisher ont été utilisés pour comparer les proportions. Une régression de Poisson a été utilisée pour comparer les taux $d$ 'incidence et estimer des tendances relatives aux maladies. La signification statistique a été considérée au degré de confiance de $95 \%$. Des analyses descriptives et inférentielles ont été menées à l'aide de Microsoft Excel 2010 et de SAS EG v. 5.1.

\section{Résultats}

\section{Aperçu}

De 2006 à 2013, les nombres totaux de cas confirmés signalés dans le Nord du Canada étaient les suivants : 270 PI, 110 SAI, $109 \mathrm{Hi}, 13 \mathrm{Ml}$ et $8 \mathrm{SBI}$ chez les nouveau-nés. Les données démographiques relatives aux cas de chaque maladie sont inscrites au tableau 1. Un total de 46 décès liés à des maladies bactériennes invasives ont été signalés.

\section{Tableau 1 : Répartitions démographiques des maladies bactériennes invasives dans le Nord du Canada par maladie, par sexe et par ethnicité, de 2006 à 2013}

\begin{tabular}{|c|c|c|c|c|c|c|}
\hline \multirow{3}{*}{$\begin{array}{l}\text { Maladie } \\
\text { (nombre } \\
\text { total) }\end{array}$} & \multirow{3}{*}{$\begin{array}{l}\text { Âge moyen, } \\
\text { années } \\
\text { (fourchette) }{ }^{1}\end{array}$} & \multirow{3}{*}{$\begin{array}{l}\text { Sexe }^{2} \text { (homme } \\
\text { ou femme) }\end{array}$} & \multicolumn{4}{|c|}{ Nombre de cas (\%) } \\
\hline & & & \multicolumn{4}{|c|}{ Ethnicité ${ }^{3}$} \\
\hline & & & $\begin{array}{l}\text { Premières } \\
\text { Nations }\end{array}$ & Inuits & Métis & $\begin{array}{c}\text { Non- } \\
\text { Autochtone }\end{array}$ \\
\hline $\mathrm{PI}(\mathrm{n}=270)$ & $39(0-92)$ & $142 / 127$ & $114(46)$ & 94 (38) & $3(1)$ & $36(15)$ \\
\hline SAI $(n=110)$ & $41(0-90)$ & $61 / 49$ & $50(48)$ & $44(42)$ & 0 & $11(10)$ \\
\hline $\mathrm{Hi}(\mathrm{n}=109)$ & $1(0-80)$ & $59 / 50$ & 28 (11) & $74(72)$ & 0 & 1 (1) \\
\hline $\mathrm{MI}(\mathrm{n}=13)$ & $0(0-56)$ & $5 / 8$ & $4(31)$ & $6(46)$ & 0 & $3(23)$ \\
\hline $\mathrm{SBI}(\mathrm{n}=8)^{\#}$ & $0(0-88)$ & $5 / 3$ & $3(38)$ & $4(50)$ & 0 & 1 (12) \\
\hline
\end{tabular}

Abréviations : $\mathrm{SBI}$, infection invasive à streptocoques du groupe $\mathrm{B} ; \mathrm{Hi}$, infection à

Haemophilus influenzae; SAl, infection invasive à streptocoques du groupe $\mathrm{A} ; \mathrm{MI}$, méningococcie invasive; PI, pneumococcie invasive

1 Deux cas dont l'âge était inconnu ont été exclus

Un cas dont le sexe était inconnu a été exclu

Trente-cinq cas dont l'ethnie était inconnue ont été exclus

Le tableau 2 montre les taux d'incidence annuels bruts des maladies dans les régions du Nord ainsi que les taux standardisés en fonction de l'âge tant pour les régions du Nord que pour le reste du Canada. À l'exception de la méningococcie invasive, les taux d'incidence standardisés en fonction de l'âge de la pneumococcie invasive, de l'infection invasive à streptocoques du groupe $\mathrm{A}$ et de l'infection à Hi étaient nettement supérieurs dans les régions du Nord.

\section{Données sur les maladies}

\section{Pneumococcie invasive (PI)}

Le taux d'incidence standardisé en fonction de l'âge (pour 100000 habitants) de pneumococcie invasive a diminué considérablement durant la période faisant l'objet du rapport $(p=0,04)$ [données non indiquées]. Les taux d'incidence standardisés en fonction de l'âge étaient semblables chez les hommes $(23,55, \mathrm{IC}: 19,65$ à 28,10$)$ et chez les femmes $(23,40, I C: 19,31$ à 28,22$)$. Le taux d'incidence annuel (pour 100000 habitants) était plus élevé chez les enfants de moins de 1 an $(132,68$, IC : 88,96 à 190,55), chez les enfants âgés de 1 à 4 ans $(49,53, I C: 35,70$ à 66,96$)$ et chez les adultes de 60 ans et plus $(47,85, I C: 35,84$ à 62,59$)$. Le taux d'incidence annuel moyen était de 29,51 (plage : 22,13 à 37,12 ) chez les habitants d'origine autochtone et de 7,57 (plage : 3,18 à 13,23) chez les habitants d'origine non autochtone : la différence est donc significative $(p<0,0001)$ [figure 1].

\section{Figure 1 : Répartition du sérotype de pneumococcie invasive par année et taux d'incidence (pour 100000 habitants) par année et ethnicité dans le Nord du Canada, de 2006 à 2013 $3^{1,2}$}

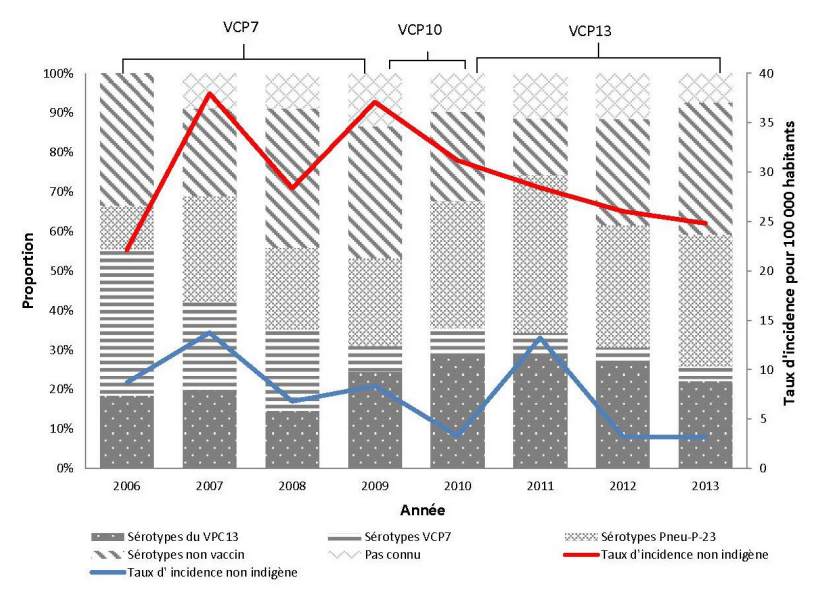

Abréviations : $\mathrm{PI}$, pneumococcie invasive; $\mathrm{VCP}$, vaccin conjugué contre le pneumocoque; VPP, vaccin polysaccharidique contre le pneumocoque

${ }^{1}$ Sérotypes du VCP7 : sept sérotypes étaient compris dans le VCP7, notamment les sérotypes 4, $6 \mathrm{~B}, 9 \mathrm{~V}, 14,18 \mathrm{C}, 19 \mathrm{~F}$ et $23 \mathrm{~F}$; sérotypes du VCP13 : six autres sérotypes étaient compris dans le VCP13, notamment les sérotypes 1, 3, 5, 6A, 7F et 19A; sérotypes du Pneu-P-23 comprenaient onze autres sérotypes par rapport au VCP13, notamment les sérotypes $2,8,9 \mathrm{~N}, 10 \mathrm{~A}, 11 \mathrm{~A}, 12 \mathrm{~F}$, $15 \mathrm{~B}, 17 \mathrm{~F}, 20,22 \mathrm{~F}$ et $33 \mathrm{~F}$

${ }^{2} \mathrm{Au}$ total, 23 cas dont les renseignements sur l'ethnicité manquaient ont été exclus du calcul du taux d'incidence

La figure 1 montre également que les répartitions proportionnelles des sérotypes de la pneumococcie invasive ont changé au fil des ans. La proportion des sérotypes du VCP7 a diminué considérablement de $37 \%(n=10)$ en 2006 à $4 \%$ $(n=1)[p=0,0004]$ en 2013. II n'y a eu aucun cas de sérotypes du VCP7 chez les enfants de moins de 2 ans depuis 2009. Parmi les cas de ce groupe, la proportion de sérotypes du VCP13 supplémentaires était de $26 \%$ avant 2011 et de $21 \%$ après 2011 : le changement n'était donc pas significatif $(p=0,49)$.

De 2006 à 2013, les sérotypes les plus courants étaient les suivants : $8(13,9 \%), 7 \mathrm{~F}$ et $19 \mathrm{~A}(6,6 \%$ chacun) ainsi que $12 \mathrm{~F}$ $(6,0 \%)$, et 3,14 et $22 \mathrm{~F}(5,4 \%$ chacun). Après 2010 , les sérotypes 
Tableau 2 : Taux d'incidence bruts et standardisés en fonction de l'âge (pour 100000 habitants) des maladies bactériennes invasives au Canada par maladie, par région et par année, de 2006 à 2013'

\begin{tabular}{|c|c|c|c|c|c|c|c|c|c|c|}
\hline \multirow{3}{*}{ Maladie } & \multicolumn{8}{|c|}{ Taux d'incidence brut } & \multicolumn{2}{|c|}{$\begin{array}{l}\text { Taux d'incidence standardisé en fonction de l'âge } \\
\text { (IC à } 95 \%)\end{array}$} \\
\hline & & & & & & & & & Régions du Nord ${ }^{2}$ & Reste du Canada ${ }^{2}$ \\
\hline & 2006 & 2007 & 2008 & 2009 & 2010 & 2011 & 2012 & 2013 & 2006 à 2013 & 2006 à 2013 \\
\hline $\mathrm{PI}$ & 18,73 & 30,97 & 23,20 & 30,42 & 20,66 & 22,96 & 16,84 & 17,35 & $23,59(20,72-26,80)$ & $8,68(8,57-8,79)$ \\
\hline SBI & 12,49 & 9,63 & 17,06 & 2,70 & 8,00 & 7,87 & 9,07 & 7,07 & $10,86(8,83-13,26)$ & $4,20(4,12-4,28)$ \\
\hline Hib & 2,78 & 0,69 & 2,05 & 0,68 & 0,00 & 0,66 & 0,65 & 0,64 & $0,89(0,45-1,71)$ & $0,09(0,08-0,10)$ \\
\hline Hi non-b ${ }^{3}$ & 7,63 & 6,88 & 2,73 & 6,76 & 11,33 & 8,53 & 7,77 & 10,92 & $8,13(6,26-10,48)^{4}$ & $0,95(0,89-1,01)^{4}$ \\
\hline MI & 3,47 & 0 & 0,68 & 0,68 & 0,67 & 1,31 & 1,30 & 0,64 & $0,87(0,46-1,63)$ & $0,55(0,52-0,58)$ \\
\hline
\end{tabular}

Abréviations : IC, intervalle de confiance; Hib, Haemophilus influenzae de type b; Hi non-b, Haemophilus influenzae d'un AUTRE type; SAI, infection invasive à streptocoques du groupe A; MI, méningococcie invasive; $\mathrm{Pl}$, pneumococcie invasive

${ }^{1}$ Deux cas d'infection à Hi invasive dont le sérotype était manquant et un cas de pneumococcie invasive dont l'âge était manquant ont été exclus du calcul du taux d'incidence

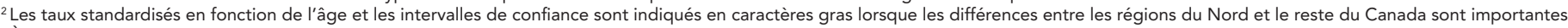

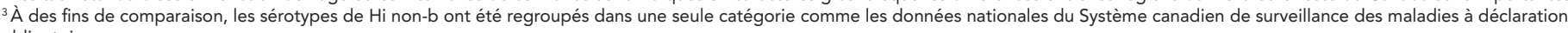
obligatoire

${ }^{4}$ Les taux d'incidence standardisés en fonction de l'âge liés à l'Hi non-b ne comprennent pas les données de 2007 et de 2008

les plus courants étaient les suivants : 7F (16,5\%), 10A (11,4\%), $19 \mathrm{~A}, 22 \mathrm{~F}$ et $33 \mathrm{~F}(7,6 \%$ chacun $)$ et $11 \mathrm{~A}(5,1 \%)$.

Des 44 cas qui avaient reçu le VCP7, les deux cas qui avaient les sérotypes du VCP7 n'avaient pas reçu tous les vaccins au moment de la maladie. Les six cas qui avaient été immunisés au moyen du VCP10 avaient des sérotypes autres que ceux du VCP10. Des 13 cas qui avaient été immunisés au moyen du VCP13, seulement un cas avait un sérotype du VCP13, et ce cas n'était pas entièrement immunisé, c'est-à-dire qu'il n'avait pas reçu les quatre doses. Des 70 cas qui avaient été immunisés au moyen du Pneu-P-23, 20 cas (29\%) avaient contracté une infection à un sérotype non vaccinal, et cinq cas (7\%) avaient un sérotype inconnu.

Au total, $87,4 \%(n=236)$ des cas de pneumococcie invasive ont été hospitalisés. Les syndromes cliniques les plus courants (tableau 3 ) étaient la pneumonie $(68,2 \%)$, la septicémie ou la bactériémie $(50,4 \%)$ et la méningite $(7,4 \%)$. Le taux de mortalité global était de $11,0 \%(n=28)$. La plupart des cas de décès concernaient des personnes âgées de 40 à 59 ans $(46,4 \%, n=13)$ ainsi que des personnes de 60 ans et plus $(35,7 \%, n=10)$. Les personnes de ces deux groupes d'âge ayant contracté une pneumococcie invasive avaient un risque de décès nettement plus élevé (taux de mortalité global de 18,1\%) que les personnes des groupes d'âge plus jeunes (taux de mortalité global de 3,9\%, $p=0,0003)$. Le taux de mortalité n'a pas varié entre les cas concernant des personnes autochtones et non autochtones $(p=0,78)$. Des 26 cas de décès dont les renseignements sur le sérotype étaient connus, la plupart avaient les sérotypes du Pneu-P-23 (46,2 \%; sérotypes du VCP13 exclus) et des sérotypes non vaccinaux $(34,6 \%)$.

\section{Infection invasive à streptocoques du groupe A (SAI)}

Le taux d'incidence annuel standardisé en fonction de l'âge de l'infection invasive à streptocoques du groupe $\mathrm{A}$ a diminué considérablement $(p=0,01)$ pendant la période du rapport. Des 110 cas d'infection invasive à streptocoques du
Tableau 3 : Manifestations cliniques courantes et résultats des cas de pneumococcie invasive, d'infection invasive à streptocoques du groupe $A, d$ 'infection à Haemophilus influenzae de type $b$, de méningococcie invasive et d'infection invasive à streptocoques du groupe B chez les nouveau-nés dans le Nord du Canada, de 2006 à $2013^{1}$

\begin{tabular}{|c|c|c|c|c|c|}
\hline \multirow{2}{*}{ Manifestations et résultats } & \multicolumn{5}{|c|}{ Nombre de cas (\%) } \\
\hline & $\begin{array}{c}\mathrm{Pl}^{2} \\
(\mathrm{n}=258)\end{array}$ & $\begin{array}{c}\text { SAI } \\
(n=106)\end{array}$ & $\begin{array}{c}\mathrm{Hi} \\
(\mathrm{n}=102)\end{array}$ & $\begin{array}{c}M l^{3} \\
(n=13)\end{array}$ & $\begin{array}{c}\mathrm{SB}^{3} \\
(n=8)\end{array}$ \\
\hline Septicémie ou bactériémie & $130(51,2)$ & $42(40,8)$ & $36(38,3)$ & 4 & 6 \\
\hline Méningite & $\begin{array}{r}19 \\
(7,5)\end{array}$ & 0 & $24(25,5)$ & 8 & 2 \\
\hline Pneumonie & $176(69,3)$ & $17(16,5)$ & $41(43,6)$ & 2 & 2 \\
\hline Empyème & $7(2,8)$ & $7(6,8)$ & $2(2,1)$ & 0 & 0 \\
\hline Arthrite purulente & $4(1,6)$ & $11(10,7)$ & $11(11,7)$ & 1 & 0 \\
\hline Fasciite nécrosante & 0 & $10(9,7)$ & 0 & 0 & 0 \\
\hline Cellulite & 0 & $33(32,0)$ & $6(6,4)$ & 0 & 0 \\
\hline Décès ${ }^{4}$ & $28(11,0)$ & $8(7,8)$ & $8(8,5)$ & $2(15,4)$ & 0 \\
\hline
\end{tabular}

Abréviations : $\mathrm{SBI}$, infection à streptocoques du groupe $\mathrm{B} ; \mathrm{Hi}$, infection à Haemophilus influenza; $\mathrm{SAl}$, infection invasive à streptocoques du groupe $\mathrm{A} ; \mathrm{MI}$, méningococcie invasive; $\mathrm{Pl}$, pneumococcie invasive

1 Pour chaque maladie, le pourcentage total de manifestations pourrait être supérieur à $100 \%$ en raison des multiples types de manifestations de chaque cas

2 Dans le terme pneumococcie invasive, on entend par "pneumonie " une pneumonie avec bactériémie

En raison du faible nombre de cas, les proportions de manifestations n'ont pas été calculées dans les cas de méningococcie invasive et d'infection à streptocoques du groupe $\mathrm{Bl}$ chez les nouveau-nés

${ }^{4}$ Les nombres totaux de cas dont les renseignements sur les résultats étaient connus sont les suivants : $254(\mathrm{PI}), 103(\mathrm{SAI}), 94(\mathrm{Hi}), 13(\mathrm{MI})$ et 7 (SBI)

groupe A, 61 étaient des hommes et 49 étaient des femmes. Les taux d'incidence standardisés en fonction de l'âge (pour 100000 habitants) des hommes $(11,86, \mathrm{IC}: 8,94$ à 15,51) étaient similaires à ceux des femmes $(9,72, I C: 7,07$ à 13,14$)$. Le taux d'incidence annuel (pour 100000 habitants) chez les enfants de moins de 1 an $(41,18$, IC : 18,83 à 78,17), chez les adultes de 60 ans et plus $(47,85, I C: 35,84$ à 62,59$)$ et chez les enfants âgés de 1 à 4 ans $(11,79, I C: 5,66$ à 21,69$)$ était le plus élevé. Le taux d'incidence annuel variait entre 2,25 et 20,44 chez les 
peuples autochtones et entre 0 et 6,80 chez les peuples non autochtones, et le taux était nettement plus élevé chez les peuples autochtones $(p<0,0001)$.

Les gènes des isolats de 74 cas de SAl étaient de type emm, et les types les plus courants étaient l'emm59 (10,8\%), I'emm1 et I'emm91 (9,5\% chacun) ainsi que l'emm41 (6,8\%). Quatre-vingtdouze pour cent $(n=101)$ des cas ont été hospitalisés. Comme le montre le tableau 3, les manifestations les plus courantes étaient la septicémie ou la bactériémie $(39,6 \%)$ et la cellulite (31,1\%). La pneumonie (16\%), l'arthrite purulente (10,4\%), la fasciite nécrosante $(9,4 \%)$ et l'empyème $(6,6 \%)$ figuraient également au nombre des manifestations fréquemment observées. Le taux de mortalité global était de 7,8 \% $(n=8)$, et tous les cas de décès (à l'exception de un cas dont l'ethnie était inconnue) concernaient des personnes autochtones. Le type de gène emm était différent dans tous les cas de décès.

Figure 2 : Répartition des sérotypes dans les cas d'infection à Haemophilus influenzae et taux d'incidence par sérotype dans le Nord du Canada par année, de 2006 à 2013 1

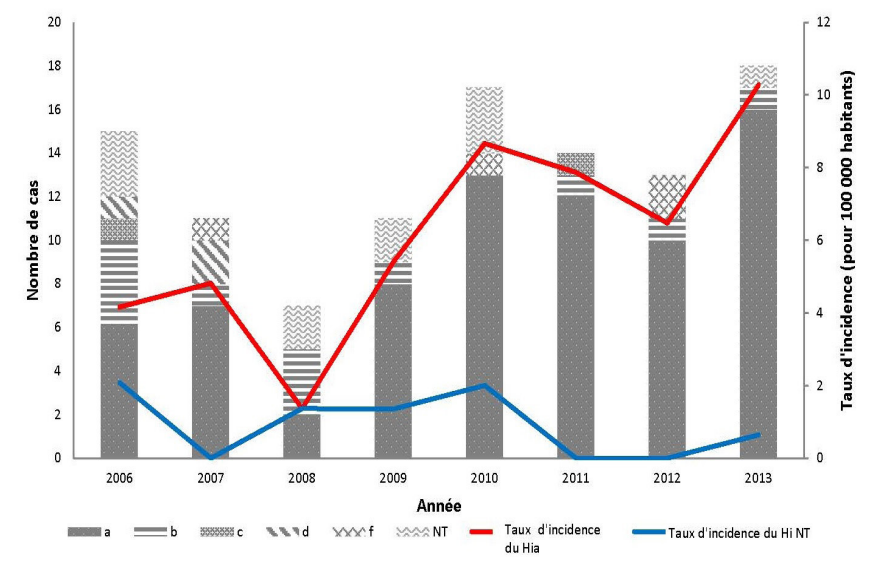

Abréviations: NT, non-typable; Hi, Haemophilus influenzae; Hi NT, Haemophilus influenzae non-typable

${ }^{1}$ Trois cas dont le sérotype était manquant ont été exclus

\section{Infection invasive à Haemophilus influenzae (Hi)}

Dans l'ensemble, il n'y a pas eu de changements significatifs dans les taux d'incidence annuels standardisés selon l'âge de I'infection à Hib $(p=0,18)$ ou de l'infection à Hi non-b $(p=0,15)$ de 2006 à 2013. Hormis six cas pour lesquels l'ethnicité était inconnue et de un cas non autochtone, l'ensemble des 102 autres cas concernaient des personnes des Premières Nations et des Inuits. Des 12 cas d'infection à Hib, 10 cas étaient âgés de moins de 18 mois, quatre cas avaient reçu tous les vaccins de la série primaire, cinq cas avaient reçu le vaccin, mais leur calendrier de vaccination n'était pas à jour, et un cas n'avait pas été immunisé.

La figure 2 montre la répartition des sérotypes des cas de Hi. Pendant la période de l'étude, l'infection à $\mathrm{Hi}$ de type a (Hia) comptait pour 69,8\% des cas, suivi de l'infection à Hib (11,3\%) et de l'infection à Hi non typable (10,4\%). Aucun cas de sérotype e n'a été signalé. Le taux d'incidence annuel (pour 100000 habitants) d'infection à Hia a augmenté considérablement $(p=0,004)$ entre 2006 et 2013 . Cinquante-trois $(71,6 \%)$ des cas d'infection à Hia concernaient des enfants âgés de moins de 5 ans. Le taux d'incidence d'infection à Hia était le plus élevé chez les enfants de moins de 1 an $(132,68$, IC : 88,86 à 190,55), suivi des enfants âgés de 1 à 4 ans $(28,31$, IC : 18,14 à 42,12$)$.

Au total, $87,5 \%(n=91)$ des cas d'infection à Hi ont été hospitalisés. Les manifestations les plus courantes (tableau 3) étaient la pneumonie $(38,7 \%)$, la septicémie ou la bactériémie $(34,0 \%)$, la méningite $(22,6 \%)$ et l'arthrite purulente $(10,4 \%)$. Le taux de mortalité global était de $8,5 \%(n=8)$, et l'infection à Hia était la cause de tous les décès.

\section{Méningococcie invasive (MI)}

Des 13 cas de méningococcie invasive, huit cas étaient de sérotype $B$ (tous âgés de moins de cinq ans), deux cas étaient de sérotype $C$ (âgés entre 40 et 59 ans) et trois cas étaient de sérotype $W$ (tous âgés de moins de 10 ans). En ce qui a trait aux manifestations, quatre cas n'avaient qu'une méningite, quatre cas avaient une méningite accompagnée d'une septicémie ou d'une bactériémie ou d'autres maladies et deux cas n'avaient qu'une septicémie ou bactériémie (tableau 3). Deux cas sont décédés; les deux étaient de sérotype $B$.

\section{Infection invasive à streptocoques du groupe $B(S B I)$ chez le nouveau-né}

Des huit cas d'infection invasive à streptocoques du groupe $B$ chez le nouveau-né, l'apparition précoce de l'infection est survenue dans six cas, et l'apparition tardive de l'infection est survenue dans deux cas. Les renseignements sur le sérotypage n'étaient disponibles que pour trois cas, dont un sérotype Ia et deux sérotypes III. La septicémie ou la bactériémie était la manifestation la plus courante $(n=6)$, suivi de la méningite $(n=2)$ et la pneumonie $(n=2)$ [tableau 3]. Aucun décès n'a été signalé.

\section{Discussion}

Au Nord du Canada, l'incidence de pneumococcie invasive, $d^{\prime}$ infection invasive à streptocoques du groupe $A$ et d'infection à Hi était de 2,6 à 10 fois plus élevée que dans le reste du Canada, particulièrement chez les peuples des Premières Nations et chez les Inuits. Ces résultats sont cohérents avec les résultats des études circumpolaires canadiennes et internationales précédentes (3-6, 21-23).

La pneumococcie invasive comptait pour la moitié des cas de maladies bactériennes invasives pendant la période de l'étude et continue d'être une cause importante de morbidité dans le Nord du Canada, particulièrement chez les nourrissons et les personnes âgées de 60 ans et plus. Le risque de décès n'a pas varié entre les peuples autochtones et les peuples non autochtones.

Le programme d'immunisation systématique des nourrissons à l'aide du VCP7 a commencé en 2002 dans certaines régions du Nord, et l'incidence de pneumococcie invasive a diminué depuis $(5,6)$. Ce rapport démontre une réduction supplémentaire et une diminution maintenue des tendances relatives à la 
pneumococcie invasive causée par un sérotype contenu dans le VCP7 ainsi que du taux d'incidence global de pneumococcie invasive. L'incidence de pneumococcie invasive causée par un sérotype contenu dans le VCP13 n'a pas changé. D'autres études longitudinales sont nécessaires pour faire une évaluation plus approfondie du VCP13. L'utilité et l'efficacité du Pneu-P-23, lequel diffère des vaccins conjugués, sont relativement plus faibles (24-26), et la protection que procure le Pneu-P-23 semble décliner après cinq ans (27). II n'est pas surprenant de voir des cas de maladie chez des personnes qui avaient été immunisées.

Depuis la mise en œuvre des programmes de vaccination contre I'infection à Hib au Canada en 1997 (8), l'infection à Hib est devenue rare dans le pays. Cependant, I'infection à Hib est toujours une préoccupation dans le Nord du Canada, car le taux de maladie est considérablement plus élevé chez les nourrissons autochtones. Certaines études suggèrent qu'une mauvaise santé, de mauvaises conditions environnementales ainsi et des logements insalubres seraient des facteurs de risque chez les enfants autochtones (28-31). L'infection à Hia a été un sérotype prédominant dans le Nord du Canada depuis le début du SISC $(5,22,32)$, tandis que l'infection à Hi non typable et de type f sont plus courantes dans les autres régions circumpolaires (32). Ce rapport démontre en outre une augmentation considérable de la prévalence de cas d'infection à Hia. Les données nationales sur les infections à $\mathrm{Hi}$ autres que de type b sont regroupées en une seule catégorie, par conséquent, les tendances et les répartitions propres à un sérotype dans le Nord du Canada ne peuvent pas être comparées à celles du reste du pays.

La méningococcie invasive est généralement rare dans le Nord du Canada ainsi que dans le reste du pays (33). Depuis la mise en œuvre des programmes d'immunisation des enfants contre le méningocoque du groupe $C$, l'incidence du méningocoque du groupe $C$ n'a jamais été aussi faible, tandis que le méningocoque du groupe B est le sérotype prédominant au Canada (33). Aucun des cas signalés pendant la période de l'étude n'aurait pu être prévenu par les programmes de vaccination à ce moment.

L'incidence d'infection invasive à streptocoques du groupe $A$ a augmenté entre 1999 et 2005 (5,32), mais elle a diminué entre 2006 et 2013. Ce changement de tendance devrait être interprété avec prudence en raison du faible nombre de cas. Les types de gène emm les plus courants étaient l'emm1, l'emm59 et l'emm91, semblables à la répartition signalée entre 1999 et 2005 (5) et pour le reste du Canada (34), mais différents des types de gènes retrouvés dans les autres régions circumpolaires, comme en Alaska, où les types de gène emm3, emm41 et emm12 étaient plus courants $(5,32)$.

En raison du manque de données sur les naissances d'enfants vivants et du très faible nombre de cas d'infection invasive à streptocoques du groupe $B$ chez les nouveau-nés, il est difficile de comparer l'épidémiologie de la maladie entre le Nord du Canada et le reste du pays ou d'autres pays.

Il est important de tenir compte des limites de l'étude dans l'interprétation des données de ce rapport. Les caractéristiques des maladies, c'est-à-dire le sérotypage, les résultats et les antécédents de vaccination, pourraient être sous-estimés ou surestimés en raison de l'insuffisance de données. Les analyses d'infections invasives à streptocoques du groupe $B$ et de méningococcies invasives ont été limitées en raison du très faible nombre de cas et du manque de données sur les naissances d'enfants vivants. En raison de l'instabilité des données causée par le faible nombre de cas et par la faible densité de population, il faut user de prudence au moment d'interpréter les résultats. Pour terminer, une analyse plus approfondie au sein des Inuits, des Premières Nations et des Métis n'a pas été possible en raison du faible nombre de données et du manque d'estimations de la population de ces groupes dans la région du SISC.

Comparativement au reste du Canada, les données indiquent que le Nord du Canada présente des taux d'incidence de pneumococcie invasive, d'infection à Hi et d'infection invasive à streptocoques du groupe A plus élevés, particulièrement chez les nourrissons et les aînés. Les groupes des Premières Nations et des Inuits sont plus vulnérables aux maladies que les peuples non autochtones. Une surveillance nationale accrue des maladies bactériennes invasives est nécessaire pour mieux comprendre les disparités en matière de maladie entre les populations du Nord du Canada et celles du reste du pays. Au Canada, le SISC est le seul système de surveillance qui recueille à la fois les données épidémiologiques et les données de laboratoires sur les maladies bactériennes invasives chez les populations nordiques. Une surveillance continue permettra de mieux comprendre l'épidémiologie des maladies et ainsi formuler des stratégies de prévention et de contrôle, notamment des recommandations en matière de vaccination pour les populations du Nord.

\section{Remerciements}

Nous souhaitons remercier tous les membres du Groupe de travail canadien sur les maladies bactériennes invasives du Système de surveillance circumpolaire internationale, particulièrement A. Mullen, B. Lefebvre, C. Cash, C. Foster, G. Tyrrell, H. Hannah, J. Proulx, K. Dehghani et Y. Jafari pour leur précieuse contribution à la surveillance du SISC et au présent rapport. Nous tenons à remercier également J. Cunliff et M. St-Jean pour la gestion des bases de données et N. Abboud pour la gestion de projet.

\section{Conflit d'intérêts}

Aucun.

\section{Financement}

La participation du Canada à la Surveillance circumpolaire internationale a été financée par l'Agence de la santé publique du Canada. 


\section{Références}

1. Parkinson AJ, Bruce MG, Zulz T. International Circumpolar Surveillance, an Arctic network for surveillance of infectious diseases. Emerg Infect Dis. 2008 Jan;14(1):18-24.

2. Vaudry W, Talling D. Invasive pneumococcal infection in first nations children in northern Alberta. Can Commun Dis Rep 2002: 28:165-72. (Disponible en français: http://www. collectionscanada.gc.ca/webarchives/20071220082651/ http://www.phac-aspc.gc.ca/publicat/ccdr-rmtc/02pdf/ cdr2820.pdf).

3. Christiansen J, Paulsen P, Ladefoged K. Invasive pneumococcal disease in Greenland. Int J Circumpolar Health 2004;63 Suppl 2:214-218.

4. Singleton R, Hammitt L, Hennessy T, Bulkow L, DeByle C, Parkinson A, et al. The Alaska Haemophilus influenzae type $b$ experience: lessons in controlling a vaccine-preventable disease. Pediatrics. 2006 Aug;118(2):e421-9.

5. Degani N, Navarro C, Deeks SL, Lovgren M. Invasive bacterial diseases in northern Canada. Emerg Infect Dis. 2008 Jan;14(1):34-40.

6. Bruce MG, Deeks SL, Zulz T, Navarro C, Palacios C, Case C, et al. Epidemiology of Haemophilus influenzae serotype a, North American Arctic, 2000-2005. Emerg Infect Dis. 2008 Jan;14(1):48-55.

7. National Advisory Committee on Immunization (NACl). Update on the invasive pneumococcal disease and recommended use of conjugate pneumococcal vaccines. Can Comm Dis Rep 2010;36(ACS-3):1-30. (Disponible en français : http://www.phac-aspc.gc.ca/publicat/ccdrrmtc/10pdf/36-acs-3.pdf)

8. Public Health Agency of Canada. Canadian Immunization Guide. Ottawa (ON). (Disponible en français : http://www. phac-aspc.gc.ca/publicat/cig-gci/index-fra.php).

9. National Advisory Committee on Immunization (NACl). An update on the invasive meningococcal disease and meningococcal vaccine conjugate recommendations. An Advisory Committee Statement (ACS). Can Commun Dis Rep 2009;36 (ACS-3):1-40. (Disponible en français : http:// www.phac-aspc.gc.ca/publicat/ccdr-rmtc/09vol35/acs-dcc-3/ index-fra.php).

10. Public Health Agency of Canada. Case definitions for communicable diseases under national surveillance. Can Commun Dis Rep 2009; 35-Suppl 2:1-123. (Disponible en français : http://www.phac-aspc.gc.ca/publicat/ccdrrmtc/09vol35/35s2/abbrev-fra.php).

11. Austrian R. The quellung reaction, a neglected microbiologic technique. Mt Sinai J Med. 1976 Nov-Dec;43(6):699-709.

12. Lovgren M, Spika JS, Talbot JA. Invasive Streptococcus pneumoniae infections: serotype distribution and antimicrobial resistance in Canada, 1992-1995. CMAJ. 1998 Feb 10;158(3):327-31.

13. Centers for Disease Control and Prevention. Streptococcus laboratory. Protocol for emm typing. Atlanta (GA): The Centers; 2015 Feb 26. http://www.cdc.gov/streplab/ protocol-emm-type.html
14. Falla TJ, Crook DW, Brophy LN, Maskell D, Kroll JS, Moxon ER. PCR for capsular typing of Haemophilus influenzae. J Clin Microbiol. 1994 Oct;32(10):2382-6.

15. Lau SK, Woo PC, Mok MY, Teng JL, Tam VK, Chan KK, et al. Characterization of Haemophilus segnis, an important cause of bacteremia, by $16 \mathrm{~S}$ rRNA gene sequencing. J Clin Microbiol. 2004 Feb;42(2):877-80.

16. Riou JY, Guibourdenche M. Laboratory methods, Neisseria and Branhamella. Paris (FR): Institut Pasteur; 1992.

17. Tsang RS, Rudolph K, Lovgren M, Bekal S, Lefebvre B, Lambertsen L, et al. International circumpolar surveillance interlaboratory quality control program for serotyping Haemophilus influenzae and serogrouping Neisseria meningitidis, 2005 to 2009. J Clin Microbiol. 2012 March;50(3):651-6.

18. Statistics Canada, Demography Division, Demographic Estimates Section, July Population Estimates, 2011 Final Intercensal Estimate. Ottawa (ON): Statistics Canada; 2015. (Disponible en français : http://www.statcan.gc.ca/pub/91215-x/91-215-x2012000-fra.pdf).

19. Statistics Canada. Table105-0503 - Health indicator profile, age-standardized rate, annual estimates, by sex, Canada, provinces and territories, occasional, CANSIM (database). Ottawa (ON): Statistics Canada; 2015. (Disponible en français : http://www5.statcan.gc.ca/cansim/pick-choisir?id= 1050503\&p2=33\&retrLang=fra\&lang $=$ fra).

20. Fay MP, Feuer EJ. Confidence intervals for directly standardized rates: a method based on the gamma distribution. Stat Med. 1997 Apr 15;16(7):791-801.

21. Helferty M, Rotondo JL, Martin I, Desai S. The epidemiology of invasive pneumococcal disease in the Canadian North from 1999 to 2010. Int J Circumpolar Health. 2013 Aug 5;72. doi:10.3402/ijch.v72i0.21606.

22. Rotondo JL, Sherrard L, Helferty M, Tsang R, Desai S. The epidemiology of invasive disease due to Haemophilus influenzae serotype a in the Canadian North from 2000 to 2010. Int J Circumpolar Health. 2013 Aug 5;72. doi:10.3402/ ijch.v72i0.21142.

23. Gounder PP, Zulz T, Desai S, Stenz F, Rudolph K, Tsang R, et al. Epidemiology of bacterial meningitis in the North American Arctic, 2000-2010. J Infect. 2015 Aug;71(2):17987.

24. Melegaro A, Edmunds WJ. The 23-valent pneumococcal polysaccharide vaccine. Part I. Efficacy of PPV in the elderly: a comparison of meta-analyses. Eur J Epidemiol. 2004;19(4):353-63.

25. Huss A, Scott P, Stuck AE, Trotter C, Egger M. Efficacy of pneumococcal vaccination in adults: a meta-analysis. CMAJ. 2009 Jan 6;180(1):48-58.

26. Leventer-Roberts M, Feldman BS, Brufman I, Cohen-Stavi CJ, Hoshen M, Balicer RD. Effectiveness of 23-valent pneumococcal polysaccharide vaccine against invasive disease and hospital-treated pneumonia among people aged $\geq 65$ years: a retrospective case-control study. Clin Infect Dis. 2015 May 15;60(10):1472-80. 
27. Andrews NJ, Waight PA, George RC, Slack MP, Miller E. Impact and effectiveness of 23-valent pneumococcal polysaccharide vaccine against invasive pneumococcal disease in the elderly in England and Wales. Vaccine. 2012 Nov 6;30(48):6802-8.

28. Banerji A, Bell A, Mills EL, McDonald J, Subbarao K, Stark $\mathrm{G}$, et al. Lower respiratory tract infections in Inuit infants on Baffin Island. CMAJ. 2001 Jun 26;164(13):1847-50.

29. Koch A, Molbak K, Homoe P, Sorensen P, Hjuler T, Olesen $M E$, et al. Risk factors for acute respiratory tract infections in young Greenlandic children. Am J Epidemiol. 2003 Aug 15;158(4):374-84.

30. Kovesi T, Creery D, Gilbert NL, Dales R, Fugler D, Thompson $B$, et al. Indoor air quality risk factors for severe lower respiratory tract infections in Inuit infants in Baffin Region, Nunavut: a pilot study. Indoor Air. 2006 Aug;16(4):266-75.

31. Banerji A, Greenberg D, White LF, Macdonald WA, Saxton A, Thomas E, et al. Risk factors and viruses associated with hospitalization due to lower respiratory tract infections in Canadian Inuit children : a case-control study. Pediatr Infect Dis J. 2009 Aug;28(8):697-701.

32. Zulz T, Bruce MG, Parkinson AJ. International circumpolar surveillance. prevention and control of infectious diseases: 1999-2008. Circumpolar Health Suppl. 2009;4:13-30.
33. Li YA, Tsang R, Desai $S$, Deehan $H$. Enhanced surveillance of invasive meningococcal disease in Canada, 2006-2011. Can Commun Dis Rep 2014;40:160-9. (Disponible en français : http://www.phac-aspc.gc.ca/publicat/ccdr-rmtc/14vol40/drrm40-09/assets/pdf/14vol40_09-fra.pdf).

34. Public Health Agency of Canada, National Microbiology Laboratory. National Laboratory Surveillance of Streptococcal Diseases In Canada - Annual Summary 2013. 2014. (Disponible en français : http://www. canadiensensante.gc.ca/publications/drugs-productsmedicaments-produits/2013-streptococcus/index-fra.php? ga=1.92842077.190996983.1435672899). 